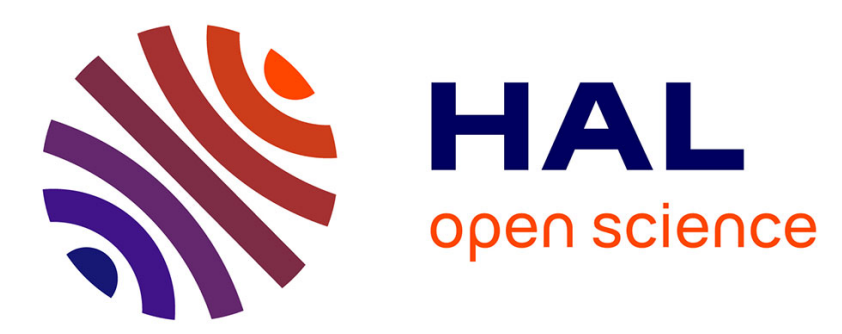

\title{
A lower bound on the expected optimal value of certain random linear programs and application to shortest paths in Directed Acyclic Graphs and reliability
}

Stéphane Chrétien, Franck Corset

\section{- To cite this version:}

Stéphane Chrétien, Franck Corset. A lower bound on the expected optimal value of certain random linear programs and application to shortest paths in Directed Acyclic Graphs and reliability. Statistics and Probability Letters, 2016, 117, pp.221-230. 10.1016/j.spl.2016.06.001 . hal-01344047

\section{HAL Id: hal-01344047 \\ https://hal.science/hal-01344047}

Submitted on 11 Jul 2016

HAL is a multi-disciplinary open access archive for the deposit and dissemination of scientific research documents, whether they are published or not. The documents may come from teaching and research institutions in France or abroad, or from public or private research centers.
L'archive ouverte pluridisciplinaire HAL, est destinée au dépôt et à la diffusion de documents scientifiques de niveau recherche, publiés ou non, émanant des établissements d'enseignement et de recherche français ou étrangers, des laboratoires publics ou privés. 


\title{
A lower bound on the expected optimal value of certain random linear programs and application to shortest paths in Directed Acyclic Graphs and reliability
}

\author{
Stéphane Chrétien ${ }^{\mathrm{a}}$, Franck Corset ${ }^{\mathrm{b}, *}$ \\ ${ }^{a}$ National Physical Laboratory, Teddington, UK \\ ${ }^{b}$ Laboratoire Jean Kuntzmann, UMR5224, Univ. Grenoble Alpes, FRANCE
}

\begin{abstract}
The paper studies the expectation of the inspection time in complex aging systems, i.e. the length of the shortest path in a Directed Acyclic Graph, with random costs on egdes. We give a lower bound for this expectation.
\end{abstract}

Keywords: Random networks, Random linear programming, Reliability, Maintenance, Dyer-Frieze-McDiarmid's inequality, Shortest path

2010 MSC: 68R10, 52B05, 90C05, 90B25

\section{Introduction and motivations}

The random shortest path problem may be a good model for describing the time to failure of very complex systems with various degradation schemes as for instance nuclear plants. In this section, we describe our motivations for studying such random shortest path problems.

\subsection{Problem statement}

Consider a complex system whose $n$ degradation states have been identified by experts. Let node 1 represent the state where the system is considered as new and let node $n$ be the state of unacceptable degradation. All maximum paths from any node of the graph end at node $n$ as in the figure below. The

\footnotetext{
${ }^{*}$ Corresponding author

Email addresses: stephane.chretien@univ-fcomte.fr (Stéphane Chrétien), franck.corset@univ-grenoble-alpes.fr (Franck Corset)
}

Preprint submitted to Statistics and Probability Letters

June 1, 2016 


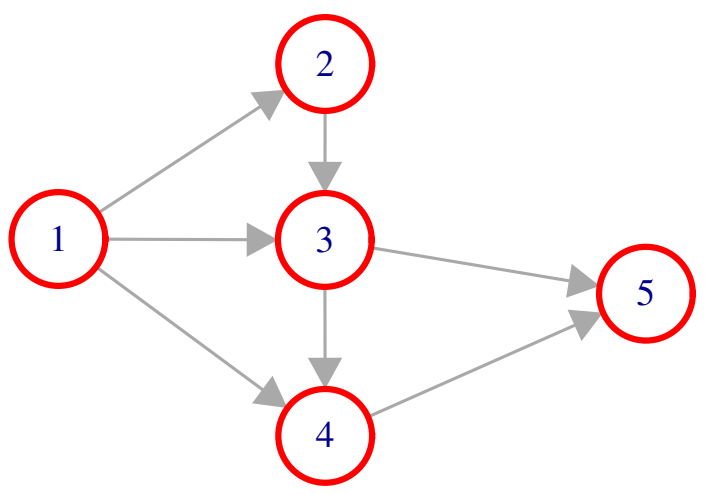

Figure 1: Graph of degradation states with Weibull transitions.

system is supposed to possibly evolve from a degradation state to any neighbor in the corresponding connected directed acyclic graph. The transition time between any two given states is assumed to follow a Weibull distribution whose parameters are estimated if the number of observations is sufficiently large. Otherwise, it is possible to make Bayesian inference in order to combine the real data with some expert opinions.

Assume we start with a brand new system. Then, evolution of the system starts in state 1. Maintenance policies require that the system be inspected before reaching state $n$, i.e. unacceptable degradation. We represent this by a connected Directed Acyclic Graph (DAG) $\mathcal{G}=(V, E)$, where $|V|=n$ and $|E|=m$. In the sequel, we assume that $m \geq n$. Such examples of complex systems have been studied in Corset (2003); Celeux et al. (2006); Adjerid et al. (2012). Moreover, Chen et al. (1999) study the shortest path, in the maintenance optimization context, for some multi-state parallel-series systems. The problem posed in this paper is to provide a lower bound on acceptable inspection times.

\subsection{Inspection times and shortest paths}

In order to simplify the analysis, we assume that evolution inside the degradation graph, a Directed Acyclic Graph (DAG), proceeds following the rule that starting from one node $i$, the system goes to state $j$ minimizing the transition 
time among neighbors of state $i$. Therefore, acceptable inspection times will be the times lower than the shortest path from state 1 to state $n$ where each edge is weighted by its transition time. In general situations, we thus may ask for

- an estimator of the expected length of the shortest path from 1 to $n$,

- a confidence interval for the expected time path.

This task is in general impossible to achieve because of the huge number of observations this should require in practice. The goal of this paper is to propose a lower bound on the expected length of the shortest path.

The paper is organized as follows. Since shortest path problems are well known to be representable as linear programs, we will address in the next section the more general problem of deriving a lower bound to the expectation of linear programs with random costs. In the third section, we specialize the study of this lower bound to an appropriate linear programming formulation of the shortest path problem. Moreover, we show that in the case of exponentially distributed random costs, the Dyer-Frieze-McDiarmid upper bound is as bad as possible. The fourth section is devoted to the application to reliability theory as motivated by the introductory example above. In particular, the Weibull distribution is proved to satisfy the assumptions under which the proposed lower bound holds.

\section{Random linear programs}

Consider the linear program with random costs given by

$$
\begin{gathered}
z=\min \quad c^{t} x \\
A x=b \\
x \geq 0
\end{gathered}
$$

where $c$ is a random vector with independent components taking values on $\mathbb{R}_{+}^{m}$, $A$ is a matrix in $\mathbb{R}^{n \times m}$ and $b$ is a vector in $\mathbb{R}^{n}$. The expectation of $c_{i}$ is denoted by $\mu_{i}$ and its variance by $\sigma_{i}^{2}$.

Throughout the paper, we will assume that the components of $c$ have a continuous density w.r.t. the Lebesgue measure. 
In the sequel, we assume that $A$ is full $\operatorname{rank}$, i.e. $\operatorname{rank}(A)=n$, and that the constraints of (1) define a polytope which is therefore a compact set. For any subset of $\{1, \ldots, m\}$, we denote by $A_{B}$ the matrix whose column set is the set of columns of $A$ indexed by $B$. We will also use the notation $x_{B}$ and $c_{B}$ for the vectors whose components are the components of $x$ and $c$ which are indexed by $B$. A set of indices $B$ is called a basis if its cardinality is $n$ and the matrix $A_{B}$ is full rank. A basis is called feasible if $x_{B}=A_{B}^{-1} b \geq 0$. A feasible basis is said to be optimal if $x^{*}$ defined by

$$
\begin{aligned}
x_{B}^{*} & =A_{B}^{-1} b \\
x_{B^{c}}^{*} & =0
\end{aligned}
$$

is an optimal solution of (1). Random linear programs have been investigated recently and many impressive results have been optained in the case of i.i.d. cost vectors. For instance, the assignment problem was investigated in Wästlund (2005), Krokhmal et al. (2007), Krokhmal and Pardalos (2009) in the asymptotic regime.

In this section, we propose a lower bound on the expected value of random linear programs in the spirit of the Dyer, Frieze and McDiarmid inequality, see Dyer et al. (1986). The Dyer-Frieze-McDiarmid inequality is a powerful tool for the analysis of some linear programming and combinatorial optimization problems with random costs, as detailed in the monograph of Steele (1997). More precisely, The Dyer-Frieze-McDiarmid bounds reads as follows.

Theorem 2.1. (Dyer-Frieze-McDiarmid) Assume that all the components of $c$ in (1) are independent and nonnegative and there exists $\beta \in(0,1]$ such that

$$
\mathbb{E}\left[c_{i} \mid c_{i} \geq h\right] \geq \mathbb{E}\left[c_{i}\right]+\beta h .
$$

Let $x$ be a feasible solution of (1). Let $z^{*}$ denote the random optimal value of (1). Then, assuming $\mathbb{E}\left[c_{1}\right] x_{1} \geq \cdots \geq \mathbb{E}\left[c_{m}\right] x_{m}$.

$$
\mathbb{E}\left[z^{*}\right] \leq \beta^{-1} \sum_{i=1}^{m} \mathbb{E}\left[c_{i}\right] x_{i}
$$


The Weibull distributions $\mathcal{W}(\eta, \gamma)$ ( $\eta$ and $\gamma$ are respectively the scale and shape parameters), has density function

$$
f(x)=\frac{\gamma}{\eta}\left(\frac{x}{\eta}\right)^{\gamma-1} \exp \left(-\left(\frac{x}{\eta}\right)^{\gamma}\right)
$$

When the edges are Weibull distributed with shape parameters $\gamma_{i}$ in the interval $[1,2]$ for $i=1, \ldots, n$, we will see in Proposition 4.1

$$
\mathrm{E}\left[c_{i} \mid c_{i} \geq h\right] \leq E\left[c_{i}\right]+\beta h
$$

with $\beta=1$. Note that Dyer-Frieze-McDiarmid requires the reverse inequality instead, in order to hold. We will however use this property to obtain a lower bound on the expectation of the optimal value of random linear programs in Theorem 2.1.

As in Dyer et al. (1986), we will need the following result which is well known to users of the simplex algorithm.

Lemma 2.1. A necessary and sufficient condition for a feasible basis to be optimal is that

$$
c_{B^{c}}^{t} \geq c_{B}^{t}\left(A_{B}^{t}\right)^{-1} A_{B^{c}}
$$

Definition 2.1. For a basis $B$, let $I_{B}$ be the index set

$$
I_{B}=\left\{i \in\{1, \ldots, n\} \mid\left(c_{B}^{t}\left(A_{B}^{t}\right)^{-1} A_{B^{c}}\right)_{i} \geq 0\right\} .
$$

Using this result and following the same reasoning as in the proof of the Dyer, Frieze and McDiarmid inequality in Dyer et al. (1986), we obtain the following proposition.

Proposition 2.1. Consider the random linear program (1) with random cost vector $c$ having independent components and satisfying (4) with $\beta \in[1,+\infty)$. Let $\mathcal{B}$ be a set of feasible bases. For each $B \in \mathcal{B}, p_{B}$ will denote the probability that $B$ is optimal. Let $\mathcal{I}_{\mathcal{B}}$ be the index set $\mathcal{I}_{\mathcal{B}}=\cap_{B \in \mathcal{B}} I_{B}$. Let $x$ be any vector satisfying the constraints of (1) and such that

$$
x_{\mathcal{I}_{\mathcal{B}}^{c}}=0 .
$$


Then, we have

$$
\mathrm{E}[z] \geq \frac{1}{\beta} \sum_{B \in \mathcal{B}} p_{B} \mathrm{E}\left[c_{B}\right] x_{B} .
$$

Proof. Fix a basis $B$ and let $E_{B}$ be the event that $B$ be optimal. Take any $x$ satisfying the primal constraints. Then we have

$$
\begin{aligned}
\mathrm{E}\left[z \mid E_{B}\right] & =\mathrm{E}\left[c_{B}^{t}\left(A_{B}\right)^{-1} b \mid E_{B}\right] \\
& =\mathrm{E}\left[c_{B}^{t}\left(A_{B}\right)^{-1}\left(A_{B} x_{B}+A_{B^{c}} x_{B^{c}}\right) \mid E_{B}\right] \\
& =\mathrm{E}\left[c_{B}^{t} x_{B}+c_{B}^{t}\left(A_{B}\right)^{-1} A_{B^{c}} x_{B^{c}} \mid E_{B}\right] .
\end{aligned}
$$

But using (4) together with (6), we have $\mathrm{E}\left[c_{B^{c}}^{t} \mid E_{B}, c_{B}\right] x_{B^{c}} \leq \mathrm{E}\left[c_{B^{c}}^{t}\right] x_{B^{c}}+$ $\beta c_{B}^{t}\left(A_{B}\right)^{-1} A_{B^{c}} x_{B^{c}}$, and thus

$$
\begin{aligned}
\mathrm{E}\left[z \mid E_{B}\right] & =\mathrm{E}\left[c_{B}^{t} \mid E_{B}\right] x_{B}+\mathrm{E}\left[\mathrm{E}\left[c_{B}^{t}\left(A_{B}\right)^{-1} A_{B^{c}} \mid E_{B}, c_{B}\right] \mid E_{B}\right] x_{B^{c}} \\
& \geq \mathrm{E}\left[c_{B}^{t} \mid E_{B}\right] x_{B}+\frac{1}{\beta}\left(\mathrm{E}\left[c_{B^{c}}^{t} \mid E_{B}\right]-\mathrm{E}\left[c_{B^{c}}^{t}\right]\right) x_{B^{c}} \\
& =\left(1-\frac{1}{\beta}\right) \mathrm{E}\left[c_{B}^{t} \mid E_{B}\right] x_{B}+\frac{1}{\beta} \mathrm{E}\left[c^{t} \mid E_{B}\right] x-\frac{1}{\beta} \mathrm{E}\left[c_{B^{c}}^{t}\right] x_{B^{c}} .
\end{aligned}
$$

Since $\beta \in[1,+\infty)$ we can rule out the term $\left(1-\frac{1}{\beta}\right) \mathrm{E}\left[c_{B}^{t} \mid E_{B}\right] x_{B}$ and using the fact that $\mathrm{E}\left[c_{B^{c}}^{t}\right] x_{B^{c}}=\mathrm{E}\left[c^{t}\right] x-\mathrm{E}\left[c_{B}^{t}\right] x_{B}$, we get

$$
\mathrm{E}\left[z \mid E_{B}\right] \geq \frac{1}{\beta} \mathrm{E}\left[c^{t} \mid E_{B}\right] x-\frac{1}{\beta}\left(\mathrm{E}\left[c^{t}\right] x-\mathrm{E}\left[c_{B}^{t}\right] x_{B}\right) .
$$

Finally take the expectation over all possible bases to obtain

$$
\begin{aligned}
\mathrm{E}[z] & \geq \frac{1}{\beta} \sum_{B \in \mathcal{B}} p_{B} \mathrm{E}\left[c^{t} \mid E_{B}\right] x-\frac{1}{\beta} \sum_{B} p_{B} \mathrm{E}\left[c^{t}\right] x+\frac{1}{\beta} \sum_{B \in \mathcal{B}} p_{B} \mathrm{E}\left[c_{B}^{t}\right] x_{B} \\
& =\frac{1}{\beta} \sum_{B} p_{B} \mathrm{E}\left[c_{B}^{t}\right] x_{B} .
\end{aligned}
$$

The result of this proposition is not completely satisfactory since the probabilities $p_{B}$ that $B$ be an optimal basis are not known. In certain cases, efficient approximations of these probabilities can be obtained using a more precise expression of $p_{B}$. Since in the case where the components of the cost vector $c$ are independent we easily get such an expression from the conditions for optimality given in Lemma 2.1. The lower bound we thus obtain is summarized in the following theorem. 
Theorem 2.2. Consider the random linear program (1) with random cost vector $c$ with independent coordinates.

a. Let $B$ be a basis for this program and for all $j \in B$ and $i \in B^{c}$, let $\alpha_{i j}=\left(\left(A_{B}^{t}\right)^{-1} A_{B^{c}}\right)_{j i}$. Then, we have

$$
p_{B}=\mathrm{E}\left[\prod_{i \in B^{c}} P\left(c_{i} \geq \sum_{j \in B} c_{j} \alpha_{j i} \mid c_{B}\right)\right] .
$$

b. Let $x$ be any vector satisfying (6) and the constraints of (1). Then

$$
E[z] \geq \frac{1}{\beta} \sum_{B \in \mathcal{B}} \mathrm{E}\left[\prod_{i \in B^{c}} P\left(c_{i} \geq \sum_{j \in B} c_{j} \alpha_{j i} \mid c_{B}\right)\right] E\left[c_{B}\right]^{t} x_{B}
$$

Proof. a. Due to independence of the components of $c$, conditionally on the value of $c_{j}, j \in B$, the events $c_{i} \geq \sum_{j \in B} c_{j} \alpha_{j i}$ are independent. Thus, the desired formula.

b. Combine a. with Proposition 2.1.

Remark 2.1. Condition (6) is easy to verify in practice by choosing a set $\mathcal{B}$ which is not too large and computing $\mathcal{I}_{\mathcal{B}}$ explicitely.

With these results in hand, we will now be able to turn to the more specialized case of random shortest paths in the next section.

\section{Random shortest paths in connected DAGs}

\subsection{Linear programming formulation}

The shortest path problem can be represented as an equivalent linear programming problem, as is well known Hoffman and Markowitz (1963). In (Papadimitriou and Steiglitz, 1998, pp. 75-79) for instance, the shortest path problem is shown to be equivalent to

$$
\min _{x \in \mathbb{R}^{n}} c_{0}^{t} x \text { subject to } A_{0} x=b \text { and } x \geq 0,
$$

where $c_{0}$ is the column vector whose components are the transition times on each edge, $A_{0}$ is the incidence matrix of the oriented degradation graph and $b$ is 
the vector $[-1,0, \cdots, 0,1]^{t}$, encoding the fact that we start the path at node 1 and end it at node $n$. Recall that the incidence matrix is constructed as follows. Its rows are indexed by the nodes of the graph while its columns are indexed by its edges with an extra column of all ones. In each column indexed by edge $(i, j)$, set the $i^{\text {th }}$ component to -1 , the $j^{\text {th }}$ component to 1 and set all other entries to zero. For instance, the incidence matrix for the graph of figure 1.1 is given by

$$
A_{0}=\left[\begin{array}{ccccccc}
-1 & -1 & -1 & 0 & 0 & 0 & 0 \\
1 & 0 & 0 & -1 & 0 & 0 & 0 \\
0 & 1 & 0 & 1 & -1 & -1 & 0 \\
0 & 0 & 1 & 0 & 1 & 0 & -1 \\
0 & 0 & 0 & 0 & 0 & 1 & 1
\end{array}\right]
$$

Any solution vector $x^{*}$ to this linear program whose components are binary, i.e. $\in\{0,1\}$ encodes a path whose edges correspond to the nonzero components of $x^{*}$. The important property is that the matrix $A_{0}$ is totally unimodular (TUM) which means that every square submatrix has determinant equal to $-1,0$ or 1 . This linear programming formulation of the problem has however a drawback: the incidence matrix is not full rank and the size of its kernel is the number of connected components of the graph, see Bollobás (1998). On the other hand, for our results to apply recall that we need the matrix $A$ in (1) to be full rank. In order to remedy this problem, we introduce the extended incidence matrix $A$, given by

$$
A=\left[A_{0} \mid e\right]
$$

where $e$ is the vector whose components are all equal to one. For instance, the extended incidence matrix for the graph of figure 1.1 is given by

$$
A=\left[\begin{array}{cccccccc}
-1 & -1 & -1 & 0 & 0 & 0 & 0 & 1 \\
1 & 0 & 0 & -1 & 0 & 0 & 0 & 1 \\
0 & 1 & 0 & 1 & -1 & -1 & 0 & 1 \\
0 & 0 & 1 & 0 & 1 & 0 & -1 & 1 \\
0 & 0 & 0 & 0 & 0 & 1 & 1 & 1
\end{array}\right]
$$


In addition, let $c$ denote the extended cost vector $\left[c_{0}^{t}, 0\right]$. Then, we get the following proposition.

Proposition 3.1. The shortest path problem is equivalent to the linear program

$$
\min _{x \in \mathbb{R}^{n}} \quad c^{t} x \text { subject to } A x=b \text { and } x \geq 0 .
$$

Proof. Let $x$ be an optimal solution of the given linear program. Then, $x$ satisfies the Karush-Kuhn-Tucker equations which are of the form:

$$
\left\{\begin{array}{l}
c+\left[\begin{array}{ll}
I & A^{t}
\end{array}\right]\left[\begin{array}{l}
u \\
v
\end{array}\right]=0, \\
A x=b \\
x \geq 0 \\
u_{i} x_{i}=0 \text { for } i=1, \ldots, n \\
u_{i} \leq 0 \\
v_{j}\left(a_{j}^{t} x-b_{j}\right)=0 \text { for } j=1, \ldots, m
\end{array}\right.
$$

where $a_{j}^{t}$ is the $j^{\text {th }}$ row of $A$ and the vectors $u \in \mathbb{R}^{n}$ and $v \in \mathbb{R}^{m}$ are the Lagrange multipliers. More precisely, the multipliers that compose the vector $u$ deal with the nonegativity constraints and the components of $v$ deal with the others. The third equation is imposed in order to select the "active" constraints at optimality. In particular, it implies that if $u_{n+1} \neq 0$ we must have $x_{n+1}=0$. On the other hand, if $u_{n+1}=0, x_{n+1}$ may be positive. In what follows, we show that $x_{n+1}$ is always null which will readily imply that this linear program also solves the shortest path problem.

Equations (16) determine a polyhedron in $\mathbb{R}^{n+1} \times \mathbb{R}^{n} \times \mathbb{R}^{m}$. Now assume that $\left[x^{t}, u^{t}, v^{t}\right]^{t}$ is a corner point of this polyhedron and that $u_{n+1}=0$. Since the matrix $A$ is now full rank, the $x$-part of the corner vector satisfies

$$
A x=b,
$$

and

$$
x_{i}=0, \quad i \in I_{x}
$$


for some index set $I_{x}$ of cardinality $n+1-m$. Now write these last nullity constraints $C x=0$ for some appropriate matrix $C$. Then by Cramer's rules, we obtain that the last coordinate $x_{n+1}$ is proportional to

$$
\operatorname{det}\left(\left[\begin{array}{c}
C \\
{\left[A_{0} \mid b\right]}
\end{array}\right]\right) .
$$

On the other hand, we know that the sum of the rows of $A$ is equal to zero and the same holds for the sum of the components of $b$. Therefore, the determinant just above is null. Therefore $x_{n+1}=0$ as announced. From this, it is easy to deduce that the vector of the first $n$ components of an optimal solution to the present linear program also solves (14).

Using this proposition, we deduce that Theorem 2.2 applies to the random shortest path problem. We now consider the Dyer-Frieze-McDiarmid bound which gives an upper bound on the expected optimal value.

\subsection{The DFM upper bound}

The upper bound for the expected optimal cost of random linear programs in Dyer et al. (1986) is a very nice result and a major contribution to the study of random optimization problems; see also Steele (1997).

It is interesting to understand to what extent the Dyer-Frieze-McDiarmid (DFM) bound is useful for the shortest path problem. Surprisingly, the answer is that the DFM bound is as bad as possible in this case, despite is remarkable efficiency on other standard combinatorial problems as shown in (Steele, 1997, Chapter 4). To understand why this happens, consider the deterministic problem where the random costs are replaced by their expected values.

$$
\begin{aligned}
\zeta= & \min E[c]^{T} x \\
& A x=b \\
& x \geq 0
\end{aligned}
$$

Now, notice that whatever the distribution of the cost vector $c$ may be, the following upper bound is immediate to obtain:

$$
\mathrm{E}[z] \leq \zeta
$$


The following proposition shows that the DFM bound is no better than this trivial upper bound.

Proposition 3.2. Consider problem (1) where the random costs are assumed to be independent and exponentially distributed. Assume that the solution of (17) is unique. Then the DFM bound is equal to the optimum value $\zeta$ of the associated deterministic program (17).

Proof. Take $x$ equal to the vector minimizing (17). It is clear that $x$ is a binary vector. It is also clear that the number of ones in this vector is less than the number of nodes in the graph. Then, the maximum value over all sets $S$ of cardinality $n$ in the right hand side term in (2) is obtained when $S$ is taken to be the set of indices $i$ for which $x_{i}=1$. Thus $\sum_{i \in S} E\left[c_{i}\right] x_{i}$ is exactly the cost of $x$, i.e. $\zeta$.

Thus, contrarily to intuition, replacing the random costs by their expected values is far from being a safe idea for the problem of providing efficient lower bounds to the mean inspection time.

Remark 3.1. Uniqueness in (17) is not restrictive since it occurs with probability one with continuous random costs.

\section{Application to reliability}

In this section, we address the problem of finding lower bounds to the inspection time of complex systems in reliability. As explained in Section 1 our main interest in studying random shortest paths problems relies in its possible application to the analysis of the time to failure for very complex systems. We will assume in this section that the transition times between two degradation states follows a Weibull distribution. In order to apply our previous results, we will need to study the Weibull distribution a little further. 


\subsection{Some properties of the Weibull distribution}

Let $X$ be a random variable with Weibull distribution $W e i b(\eta, \gamma)$, i.e. with probability density function given by

$$
f_{X}(t)=\frac{\gamma}{\eta}\left(\frac{t}{\eta}\right)^{\gamma} e^{-\left(\frac{t}{\eta}\right)^{\gamma}}
$$

Then, the mean residual time to failure (MRTF) is given by

$$
G_{X}(h)=E[X \mid X \geq h]=\eta e^{\left(\frac{h}{\eta}\right)^{\gamma}} \Gamma\left(1+\frac{1}{\gamma},\left(\frac{h}{\eta}\right)^{\gamma}\right),
$$

where $\Gamma(a, h)$ is the incomplete gamma function defined by

$$
\Gamma(a, h)=\int_{h}^{+\infty} t^{a-1} e^{-t} d t .
$$

Lemma 4.1. Let $X$ be a Weibull, denoted $W e i b(\eta, \gamma)$, distributed random variable. Then,

a. the first two derivatives of the MRTF for a Weibull distributed variable $X$ are given by

$$
G_{X}^{\prime}(h)=\gamma\left(\frac{h}{\eta}\right)^{\gamma}\left(\frac{\eta}{h} e^{\left(\frac{h}{\eta}\right)^{\gamma}} \Gamma\left(1+\frac{1}{\gamma},\left(\frac{h}{\eta}\right)^{\beta}\right)-1\right)
$$

and

$$
\begin{aligned}
G_{X}^{\prime \prime}(h)= & -\frac{\gamma^{2}}{\eta^{\gamma}} h^{(\gamma-1)}\left(1+\gamma\left(\frac{h}{\eta}\right)^{\gamma}\right)+ \\
& \frac{\gamma}{\eta^{(\gamma-1)}} h^{(\gamma-2)} e^{\left(\frac{h}{\eta}\right)^{\gamma}} \Gamma\left(1+\frac{1}{\gamma},\left(\frac{h}{\eta}\right)^{\gamma}\right)\left(\gamma\left(\frac{h}{\eta}\right)^{\gamma}+\gamma-1\right) .
\end{aligned}
$$

Moreover,

b. when $\gamma \geq 1$ we have

$$
\lim _{h \rightarrow 0} G_{X}^{\prime}(h)=0, \quad \lim _{h \rightarrow+\infty} G_{X}^{\prime}(h)=1 \text { and } \forall h \geq 0, \quad G_{X}^{\prime \prime}(h)<1 .
$$

Proof. a. We omit the proof of the formula for the first and second derivative of $G_{X}$.

b. Now, since $G_{X}^{\prime}$ is clearly continuous on $\mathbb{R}^{+}$and $G_{X}^{\prime}(0)=0$, we obtain the first assertion in $\mathrm{b}$. Using the transformation $u=t-(h / \eta)^{\gamma}$, the first of the two terms between parenthesis in (22) can we written

$$
\eta e^{\left(\frac{h}{\eta}\right)^{\gamma}} \Gamma\left(1+\frac{1}{\gamma},\left(\frac{h}{\eta}\right)^{\gamma}\right)=h \int_{0}^{+\infty} e^{-u}\left(1+\left(\frac{\eta}{h}\right)^{\gamma} u\right)^{\frac{1}{\gamma}} d u .
$$


For all $u \geq 0$ we have the following Taylor expansion

$$
\left(1+\left(\frac{\eta}{h}\right)^{\gamma} u\right)^{\frac{1}{\gamma}}=1+\frac{1}{\gamma} \frac{\eta^{\gamma}}{h^{\gamma}} u+o\left(\frac{1}{h^{\gamma}}\right) .
$$

Multiplying by $e^{-u}$ and integrating, we obtain

$$
\begin{aligned}
\int_{0}^{+\infty} e^{-u}\left(1+\left(\frac{\eta}{h}\right)^{\gamma} u\right)^{\frac{1}{\gamma}} d u & =\int_{0}^{+\infty} e^{-u} d u+\frac{1}{\gamma} \frac{\eta^{\gamma}}{h^{\gamma}} \int_{0}^{+\infty} e^{-u} u d u+o\left(\frac{1}{h^{\gamma}}\right) \\
& =1+\frac{1}{\gamma} \frac{\eta^{\gamma}}{h^{\gamma}}+o\left(\frac{1}{h^{\gamma}}\right) .
\end{aligned}
$$

Combining with (22), we deduce from a simple analysis of the second derivative that $\lim _{h \rightarrow+\infty} G_{X}^{\prime}(h)=1$ and $G_{X}^{\prime}(h) \leq 1$ for all $h \in \mathbb{R}_{+}$.

\subsection{The lower bound}

In this subsection, we work out an easily computable lower bound derived from Theorem 2.2. We first have the following crucial result saying that the most commonly encountered Weibull distributions in reliability theory satisfy the main assumption of Proposition 2.1 and Theorem 2.2.

Proposition 4.1. Assume that $X$ has distribution Weib $(\eta, \gamma)$ with $\gamma>1$. Then, for all $h \geq 0$, we have

$$
E[X \mid X \geq h] \leq E[X]+h .
$$

Proof. This is a direct consequence of lemma 4.1.

In the next theorem, we derive an explicit lower bound from Theorem 2.2 in the case of Weibull distributions.

Theorem 4.1. Consider the random linear program (1) with random cost vector $c$ with independent components and assume that each component $c_{i}, 1 \leq i \leq$ $n$ follows a Weibull distribution Weib $\left(\eta_{i}, \gamma_{i}\right)$

a. Let $B$ be a basis for this program and for all $j \in B$ and $i \in B^{c}$, let $\alpha_{i j}=\left(\left(A_{B}^{t}\right)^{-1} A_{B^{c}}\right)_{j i}$. Then, we have

$$
p_{B} \geq 1-\sum_{i \in B^{c}} \frac{4 \sqrt{e}\left(\sum_{j \in B} \alpha_{j i}^{2} \eta_{j}^{2} \Gamma\left(1+\frac{2}{\gamma_{j}}\right)\right)^{\frac{1}{2}}+\left|\sum_{j \in B} \alpha_{j i} \eta_{j} \Gamma\left(1+\frac{1}{\gamma_{j}}\right)\right|}{\eta_{i}^{\gamma_{i}}} .
$$


b. Let $x$ be any vector satisfying (6) and the constraints of (1). Then

$E[z] \geq \sum_{B \in \mathcal{B}}\left(1-\sum_{i \in B^{c}} \frac{4 \sqrt{e}\left(\sum_{j \in B} \alpha_{j i}^{2} \eta_{j}^{2} \Gamma\left(1+\frac{2}{\gamma_{j}}\right)\right)^{\frac{1}{2}}+\left|\sum_{j \in B} \alpha_{j i} \eta_{j} \Gamma\left(1+\frac{1}{\gamma_{j}}\right)\right|}{\eta_{i}^{\gamma_{i}}}\right) E\left[c_{B}\right]^{t} x_{B}$.

Proof. a. Theorem 2.2.a. gives the following formula for $p_{B}$ :

$$
p_{B}=\mathrm{E}\left[\prod_{i \in B^{c}} \exp -\left(\max \left\{0, \frac{\sum_{j \in B} \alpha_{j i} c_{j}}{\eta_{i}}\right\}\right)^{\gamma_{i}}\right],
$$

where the expectation is taken with respect to the variables $c_{j}, j \in B$. Now since $\exp (-x) \geq 1-x$, we obtain that

$$
\begin{aligned}
p_{B} & \geq \mathrm{E}\left[1-\sum_{i \in B^{c}} \max \left\{0,\left(\frac{\sum_{j \in B} \alpha_{j i} c_{j}}{\eta_{i}}\right)^{\gamma_{i}}\right\}\right], \\
& =1-\sum_{i \in B^{c}} \mathrm{E}\left[\max \left\{0, \frac{\sum_{j \in B} \alpha_{j i} c_{j}}{\eta_{i}}\right\}^{\gamma_{i}}\right] .
\end{aligned}
$$

Thus,

$$
p_{B} \geq 1-\sum_{i \in B^{c}} \frac{\mathrm{E}\left[\max \left\{0, \sum_{j \in B} \alpha_{j i} c_{j}\right\}^{\gamma_{i}}\right]}{\eta_{i}^{\gamma_{i}}} .
$$

In order to simplify the subsequent computations, we will use the crude majorization:

$$
\max \left\{0, \sum_{j \in B} \alpha_{j i} c_{j}\right\} \leq\left|\sum_{j \in B} \alpha_{j i} c_{j}\right|
$$

which gives

$$
p_{B} \geq 1-\sum_{i \in B^{c}} \frac{\mathrm{E}\left[\left|\sum_{j \in B} \alpha_{j i} c_{j}\right|^{\gamma_{i}}\right]}{\eta_{i}^{\gamma_{i}}} .
$$

Now, our next goal is to use the Kintchine inequalities in order to bound the last expression by a quantity expressed in terms of the $l_{2}$ norm which will be easier to control. For this purpose, one might want to center the random variables involved in (33) and use the triangle inequality to obtain

$$
\begin{aligned}
\mathrm{E}\left[\left|\sum_{j \in B} \alpha_{j i} c_{j}\right|^{\gamma_{i}}\right]^{\frac{1}{\gamma_{i}}} & =\mathrm{E}\left[\left|\sum_{j \in B} \alpha_{j i}\left(c_{j}-\mathrm{E}\left[c_{j}\right]+\mathrm{E}\left[c_{j}\right]\right)\right|^{\gamma_{i}}\right]^{\frac{1}{\gamma_{i}}} \\
& \leq \mathrm{E}\left[\left|\sum_{j \in B} \alpha_{j i}\left(c_{j}-\mathrm{E}\left[c_{j}\right]\right)\right|^{\gamma_{i}}\right]^{\frac{1}{\gamma_{i}}}+\mathrm{E}\left[\left|\sum_{j \in B} \alpha_{j i} \mathrm{E}\left[c_{j}\right]\right|^{\gamma_{i}}\right]^{\frac{1}{\gamma_{i}}} \\
& =\mathrm{E}\left[\left|\sum_{j \in B} \alpha_{j i}\left(c_{j}-\mathrm{E}\left[c_{j}\right]\right)\right|^{\gamma_{i}}\right]^{\frac{1}{\gamma_{i}}}+\left|\sum_{j \in B} \alpha_{j i} \mathrm{E}\left[c_{j}\right]\right|
\end{aligned}
$$


Using Jensen's inequality, a standard trick gives

$$
\begin{aligned}
\mathrm{E}\left[\left|\sum_{j \in B} \alpha_{j i}\left(c_{j}-\mathrm{E}\left[c_{j}\right]\right)\right|^{\gamma_{i}}\right]^{\frac{1}{\gamma_{i}}} & =\mathrm{E}\left[\left|\sum_{j \in B} \alpha_{j i}\left(c_{j}-\mathrm{E}\left[c_{j}^{\prime}\right]\right)\right|^{\gamma_{i}}\right]^{\frac{1}{\gamma_{i}}} \\
& \leq \mathrm{E}\left[\left|\sum_{j \in B} \alpha_{j i}\left(c_{j}-c_{j}^{\prime}\right)\right|^{\gamma_{i}}\right]^{\frac{1}{\gamma_{i}}}
\end{aligned}
$$

where $c_{j}^{\prime}, j \in B$ are i.i.d. variables independent of $c_{j}, j \in B$ and such that $c_{j}$ has same distribution as $c_{j}^{\prime}, j \in B$. Let $\epsilon_{j}, j \in B$ be standard Rademacher \pm 1 random variables. Since $\sum_{j \in B} \alpha_{j i}\left(c_{j}-c_{j}^{\prime}\right)$ has the same distribution as $\sum_{j \in B} \alpha_{j i} \epsilon_{j}\left(c_{j}-c_{j}^{\prime}\right)$, we have

$$
\begin{aligned}
\mathrm{E}\left[\left|\sum_{j \in B} \alpha_{j i}\left(c_{j}-\mathrm{E}\left[c_{j}\right]\right)\right|^{\gamma_{i}}\right]^{\frac{1}{\gamma_{i}}} & =\mathrm{E}\left[\left|\sum_{j \in B} \alpha_{j i} \epsilon_{j}\left(c_{j}-c_{j}^{\prime}\right)\right|^{\gamma_{i}}\right]^{\frac{1}{\gamma_{i}}} \\
& \leq 2 \mathrm{E}\left[\left|\sum_{j \in B} \alpha_{j i} \epsilon_{j} c_{j}\right|^{\gamma_{i}}\right]^{\frac{1}{\gamma_{i}}}
\end{aligned}
$$

where we used once again the triangle inequality. Notice that

$$
\begin{aligned}
\mathrm{E}\left[\left|\sum_{j \in B} \alpha_{j i} \epsilon_{j} c_{j}\right|^{\gamma_{i}}\right]^{\frac{1}{\gamma_{i}}} & =\mathrm{E}\left[\mathrm{E}\left[\left|\sum_{j \in B} \alpha_{j i} \epsilon_{j} c_{j}\right|^{\gamma_{i}} \mid c_{j}, j \in B\right]\right]^{\frac{1}{\gamma_{i}}} \\
& =\mathrm{E}\left[\left(\mathrm{E}\left[\left|\sum_{j \in B} \alpha_{j i} \epsilon_{j} c_{j}\right|^{\gamma_{i}} \mid c_{j}, j \in B\right]^{\frac{1}{\gamma_{i}}}\right)^{\gamma_{i}}\right]^{\frac{1}{\gamma_{i}}} .
\end{aligned}
$$

On the other hand, Khintchine's inequality gives

$$
\mathrm{E}\left[\left(\mathrm{E}\left[\left|\sum_{j \in B} \alpha_{j i} \epsilon_{j} c_{j}\right|^{\gamma_{i}} \mid c_{j}, j \in B\right]^{\frac{1}{\gamma_{i}}}\right)^{\gamma_{i}}\right]^{\frac{1}{\gamma_{i}}} \leq C_{\gamma_{i}} \mathrm{E}\left[\left(\left(\sum_{j \in B} \alpha_{j i}^{2} c_{j}^{2}\right)^{\frac{1}{2}}\right)^{\gamma_{i}}\right]^{\frac{1}{\gamma_{i}}}
$$

where $C_{\gamma_{i}}$ is equal to $\sqrt{2 e \gamma_{i}}$ in the present context. Thus,

$$
\mathrm{E}\left[\left(\mathrm{E}\left[\left|\sum_{j \in B} \alpha_{j i} \epsilon_{j} c_{j}\right|^{\gamma_{i}} \mid c_{j}, j \in B\right]^{\frac{1}{\gamma_{i}}}\right)^{\gamma_{i}}\right]^{\frac{1}{\gamma_{i}}} \leq \sqrt{2 e \gamma_{i}}\left(\mathrm{E}\left[\left(\sum_{j \in B} \alpha_{j i}^{2} c_{j}^{2}\right)^{\frac{\gamma_{i}}{2}}\right]^{\frac{2}{\gamma_{i}}}\right)^{\frac{1}{2}} .
$$

Moreover, since $p \mapsto E\left[|X|^{p}\right]^{\frac{1}{p}}$ is an increasing function and $\gamma_{i}$ is assumed to belong to $[1,2]$, we obtain the simpler bound

$$
\begin{aligned}
\mathrm{E}\left[\left(\mathrm{E}\left[\left|\sum_{j \in B} \alpha_{j i} \epsilon_{j} c_{j}\right|^{\gamma_{i}} \mid c_{j}, j \in B\right]^{\frac{1}{\gamma_{i}}}\right)^{\gamma_{i}}\right]^{\frac{1}{\gamma_{i}}} & \leq 2 \sqrt{e} \mathrm{E}\left[\sum_{j \in B} \alpha_{j i}^{2} c_{j}^{2}\right]^{\frac{1}{2}} \\
& =2 \sqrt{e}\left(\sum_{j \in B} \alpha_{j i}^{2} \mathrm{E}\left[c_{j}^{2}\right]\right)^{\frac{1}{2}}
\end{aligned}
$$


Moreover, since $\mathrm{E}\left[c_{j}^{2}\right]=\eta_{j}^{2} \Gamma\left(1+\frac{2}{\gamma_{j}}\right)$, we have

$$
\begin{aligned}
\mathrm{E}\left[\left(\mathrm{E}\left[\left|\sum_{j \in B} \alpha_{j i} \epsilon_{j} c_{j}\right|^{\gamma_{i}} \mid c_{j}, j \in B\right]^{\frac{1}{\gamma_{i}}}\right)^{\gamma_{i}}\right]^{\frac{1}{\gamma_{i}}} & \leq 2 \sqrt{e} \mathrm{E}\left[\sum_{j \in B} \alpha_{j i}^{2} c_{j}^{2}\right]^{\frac{1}{2}} \\
& =2 \sqrt{e}\left(\sum_{j \in B} \alpha_{j i}^{2} \eta_{j}^{2} \Gamma\left(1+\frac{2}{\gamma_{j}}\right)\right)^{\frac{1}{2}} .
\end{aligned}
$$

Combining this result with (33), (34), (36), (37), and replacing $\mathrm{E}\left[c_{j}\right]=\eta_{j} \Gamma(1+$ $\left.\frac{1}{\gamma_{j}}\right)$ in (34), we finally obtain the desired result.

b. This follows from part a. and Proposition 2.1.

\section{Conclusion and perspectives}

In this paper, we derived a lower bound (Theorem 4.1) on the probability that a given path is optimal for the shortest path problem with independent arc weights with Weibull distributions. For this purpose, we used the linear programming formulation of the problem and extended the work of Dyer et al. (1986) (Theorem 2.2).

The results presented here are of a theoretical nature. Further refinements and applications to real data will be proposed in a subsequent paper.

\section{References}

S. Adjerid, T. Aggad, and D. Benazzouz. Performance evaluation and optimisation of industrial system in a dynamic maintenance. American journal of Intelligent Systems, 2(5):82-92, 2012.

Béla Bollobás. Modern graph theory, volume 184. Springer Science \& Business Media, 1998.

G. Celeux, F. Corset, A. Lannoy, and B. Ricard. Designing a bayesian network for preventive maintenance from expert opinions in a rapid and reliable way. Reliability Engineering and System Safety, 91(7):849-856, 2006. 
Cheng Chen, Max Q-H Meng, and Ming J Zuo. Selective maintenance optimization for multi-state systems. In Electrical and Computer Engineering, 1999 IEEE Canadian Conference on, volume 3, pages 1477-1482. IEEE, 1999.

F. Corset. Maintenance optimization from Bayesian networks and reliability with doubly censored data. Thèse Université Joseph Fourier, Grenoble, 2003.

M.E. Dyer, A.M. Frieze, and C.J.H. McDiarmid. On linear programs with random costs. Math. Programming, 35(1):3-16, 1986.

AJ Hoffman and HM Markowitz. A note on shortest path, assignment, and transportation problems. Naval Research Logistics Quarterly, 10(1):375-379, 1963.

Pavlo A Krokhmal and Panos M Pardalos. Random assignment problems. European Journal of Operational Research, 194(1):1-17, 2009.

Pavlo A Krokhmal, Don A Grundel, and Panos M Pardalos. Asymptotic behavior of the expected optimal value of the multidimensional assignment problem. Mathematical programming, 109(2-3):525-551, 2007.

C. H. Papadimitriou and K. Steiglitz. Combinatorial Optimization: Algorithms and Complexity. Dover, 1998.

J Michael Steele. Probability theory and combinatorial optimization, volume 69 . Siam, 1997.

Johan Wästlund. A proof of a conjecture of buck, chan, and robbins on the expected value of the minimum assignment. Random Structures $\&$ Algorithms, 26(1-2):237-251, 2005. 